\title{
Nemico e propaganda
}

\section{Andrea Baravelli}

Storicamente, 1 (2005).

ISSN: 1825-411X. Art. no. 13. DOI: 10.12977/stor518

La figura del nemico ha sempre rappresentato un elemento indispensabile per il buon funzionamento dei sistemi di propaganda. Insomma, si tratta di un protagonista assoluto - se non unico - dell'argomentazione di tipo propagandistico; una figura dalla rilevanza tale da costringere l'intero spazio della politica a organizzarsi in sua funzione. II valore in termini di forza conativa, di capacità di spingere all'azione, non era certo sottovalutato dagli antichi, tuttavia fu solo con la Rivoluzione francese che fu compresa quale importanza potesse avere l'evocare lo spettro del nemico quale elemento di volta di un moderno sistema propagandistico. Gli uomini della Rivoluzione, in particolare, non solo scoprirono quali fossero i modi migliori per fare del nemico un tema di propaganda adeguato, ma intuirono il nesso esistente fra efficacia del messaggio propagandistico e azione volta a provocare forti emozioni nelle masse popolari. Insomma, con la Rivoluzione francese si spezzò la relazione fino a quel momento obbligata fra persuasione e razionalità. Senza studi di psicologia sociale alle loro spalle, gli uomini incaricati di promuovere la Rivoluzione compresero tuttavia come il ricorso alle emozioni - in particolare, al sentimento della paura - potesse costituire un irrinunciabile atout per radicare la Rivoluzione stessa nell'animo delle popolazioni. Una volta definiti i lineamenti del nemico, infatti, si sarebbe potuto ottenere l'adesione di quelle stesse masse su questioni anche molto differenti rispetto alla semplice necessità della lotta per opporvisi. L'effetto della designazione di un nemico per l'opinione pubblica è infatti triplice. Da una parte essa conduce alla cristallizzazione della fedeltà dell'opinione 
pubblica a un dato progetto politico (infatti, individuando un nemico non solo si orienta tale opinione pubblica contro qualcuno, ma la si sollecita anche a provare un senso di gratitudine nei confronti di chi quel nemico ha scoperto e denunciato). Da un'altra, il concentrare il risentimento della collettività nei confronti di un nemico equivale a "compattare" quella stessa comunità con il pretesto dell'esistenza di un elemento irriducibile e pericoloso. Infine, il definire un nemico dona al potere la possibilità di deviare il risentimento popolare che, altrimenti, investirebbe il potere stesso. Insomma, classificare l'altro da sé secondo la categoria di nemico piuttosto che secondo quella di amico significa indicare la strada che si vuole sia seguita dall'intera comunità. A causa del suo inscriversi all'interno di un doppio movimento l'affermarsi dell'ideologia quale origine e determinante dell'agire politico, da una parte; l'impetuoso sviluppo della società di massa e del progresso tecnologico, dall'altra -, il Novecento può ben essere definito come il secolo della propaganda. Anzi, il secolo del nemico assoluto. Un nemico costruito, nei lineamenti più minuti come nel senso della pericolosità, dal politico attraverso la propaganda. Dopo la Grande guerra, prima importante prova, la propaganda si perfezionò all'interno dei regimi totalitari. L'asprezza ideologica della guerra fredda, poi, s'incaricò di confermare l'importanza della figura del nemico quale perno dell'intero sistema di rappresentazione della politica e dell'esistenza. La "fine delle ideologie" ha forse mutato il quadro di riferimento? L'esperienza degli ultimi anni pare svolgersi nel segno della continuità: nelle società contemporanee, caratterizzate da molteplici flussi d'informazione e dalla sempre maggiore incapacità di ricondurre in termini di comprensibilità la complessità dell'esistente, l'uso della categoria del nemico rimane indispensabile poiché fornisce una chiave ai fini della ricomposizione di una realtà frammentata e apparentemente incongruente. 